\title{
The Chaotic Structure Of The \$C/\$US Exchange Rate
}

Rae Weston, (E-mail: Rae.weston@mgsm.edu.au), Macquarie Graduate School of Management, Australia Prem Premachandran, Macquarie Graduate School of Management, Australia

\begin{abstract}
This paper provides a brief survey of previous applications of chaotic analysis to exchange rate series and of the connection between theoretical models of exchange rates and the chaotic approach. and then considers the key elements required to establish a chaotic structure and apply the analysis to the daily \$C/\$US exchange rates over the period 1973 to 2003 In particular the overshooting hypothesis as represented by the chaotic structure is investigated.
\end{abstract}

\section{THE CHAOTIC STRUCTURE OF THE \$C/\$US EXCHANGE RATE}

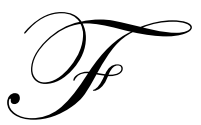

or open economies such as Canada the exchange rate should be regarded as the most significant asset price. Analysis of the structure of this asset price therefore has more than usual interest. Canada had a flexible exchange rate system between 1950 and 1962. It returned to the system in June1970 and has persisted with the system ever since. This paper investigates the overshooting hypothesis for exchange rates as represented by the chaotic structure of the \$C/\$US exchange rate for the period 1973 to 2003.

In the next section we discuss the exchange rate regime in Canada over this period and then move in the following section to consider the previous analyses of the Canadian exchange rate. We discuss the overshooting hypothesis and the tests for chaotic structure applied before reporting our results and considering their implications.

\section{THE EXCHANGE RATE REGIME IN CANADA}

Laidler (1999) notes that an exchange rate and its regime are part of an economy's coherent monetary order and identifies three separate monetary orders in Canada. These are 1970-75 when there was no well-defined anchor; 1975-1981 when a target growth rate for M1 was set; and 1981-1991 when there was an increasing emphasis on price stability. Laidler describes Canada's current monetary order as defined by inflation-control targets.

Gordon Theissen, Governor of the Bank of Canada (2000) in an address entitled "Why a floating exchange rate regime makes sense for Canada" argued that "The real value of a floating exchange rate for Canada is that it allows us to have different monetary conditions from the United States-monetary conditions appropriate to our own economic circumstances, even as we pursue the same objective of low inflation. The significance of having this option is our ability to respond to external economic shocks that affect us differently from our southern neighbours, or to respond to differences in domestic economic policies" (at p.3).

It is widely recognised that the term "floating" with respect to an exchange rate does not imply an entirely free float. The Bank of Canada conducts foreign exchange intervention in the promotion of orderly markets for the Canadian dollar. The mechanisms by which sterilized intervention may affect an exchange rate are the portfolio balance approach; the liquidity approach; the signalling approach and the noise trading approach. The portfolio balance approach suggests to the extent that foreign and domestic securities are imperfect substitutes that intervention could affect the level of the exchange rate by changing the supply of foreign and domestic securities. This has received little empirical support. The liquidity approach suggests that if the amount of intervention is large relative to the market turnover for a short time it may provide liquidity and have a flow-driven impact on the currency (Beattie and Fillion (1999) at p. 1). The signalling approach relies on the perhaps specious assumption that that the central 
bank possesses better information than the market. The literature on this issue especially Dominguez (1998) is that intervention does not necessarily convey clear signals While it might be expected that central bank intervention would render statistical analysis of the structure of the exchange rate impossible, the evidence of Murray, Zelmer and McManus (1996) and Beattie and Fillion (1999) is that at best there may have been a small stabilizing effect of expected intervention while non-discretionary intervention has not affected volatility. D'Souza (2002) recognises that the optimal strategy of each foreign exchange dealer in the market is to "amplify any customer trades in interdealer trading" and suggests that intervention to reduce volatility may have the opposite effect.

\section{PREVIOUS TESTS OF STRUCTURE}

Tests for excessive volatility and speculative bubbles undertaken by Murray, van Norden and Vigfusson (1996) suggested that most of the swings in the Canadian dollar over the 1975-95 period may be accounted for by fundamental factors such as commodity prices and the terms of trade. While Canada's foreign trade is dominated by the United States, the composition of output is significantly different between the two economies.

On the basis of the evidence discussed above there is a reasonable expectation that a statistical investigation of the structure of the Canadian dollar's moves over the period 1970-2002 might uncover a result Previous tests of structure .

Tests for excessive volatility and speculative bubbles undertaken by Murray, van Norden and Vigfusson (1996) suggested that most of the swings in the Canadian dollar over the 1975-95 period may be accounted for by fundamental factors such as commodity prices and the terms of trade. If we consider the discussions of the movements in the Canadian exchange rate (i.e.LaFrance and van Noreden, etc) the recipe for chaos described by Da Silva (1999at p.2) "Starting from an initial deviation a given endogenous variable perpetually fluctuates around equilibrium. As the deviations become too large, the centrifugal force brings the variable back towards equilibrium, while once back to the neighbourhood of equilibrium, the centrifugal force forces it to diverge again." seems consistent. Powell's description of the Canadian dollar's movements in the 1970s, 1980s and 1990s refer to weakness, recovery, rebounding, and depreciating which may also be seen to be interpretable using the "recipe".

Murray, van Norden and Vigfusson (1996) found using a Markov-switching model that there were long periods when noise traders or technical trades appeared to dominate the exchange market, although in their view these periods were relatively stable. . The noise-trading approach suggested by Hung (1997) attempts to alter the technical outlook for the currency by increasing or decreasing volatility.

\section{THE OVERSHOOTING HYPOTHESIS}

Empirical research on nominal exchange rates has shown that most changes in exchange rates are unexpected and actual exchange rate movement appear to overshoot movements in the equilibrium exchange rate (Bask, 2002). One explanation for exchange rate volatility is speculation. MIT economist Dornbusch (1976) argued that even without speculation, exchange rates will be highly volatile because of a phenomenon that he labelled "overshooting." According to his theory, a monetary expansion will induce an immediate depreciation in the exchange rate in the short run and accounts therefore for the fluctuations in the exchange rate and the term of trade. If real output is fixed, the monetary expansion will lower interest rates and cause the exchange rate to overshoot its long-run depreciation. If output responds to aggregate demand then the interest rate changes and exchange rate changes will be dampened.

Let us consider the "overshooting" hypothesis in the context of chaotic analysis. Stewart (1997 in Chapter 8) describes the "recipe" for chaos as deriving from the interaction of "stretching" and "folding". Da Silva (1999, p.2) describes the same process as "starting from an initial deviation a given endogenous variable perpetually fluctuates around equilibrium. As the deviations become too large, the centrifugal force brings the variable back towards equilibrium, while once back to the neighbourhood of equilibrium, the centrifugal force forces it to diverge again." 
In terms of exchange rate movements this would suggest that overshooting occurs as a consequence of the centrifugal force which is in the end constrained by the centripedal force which recalls the exchange rate back towards equilibrium. In context of mathematics of chaotic behaviour, a positive Lyapunov exponent measures the exponential divergence of nearby points (centrifugal force) and a negative exponent measures the exponential convergence (centripedal force). Two trajectories on the chaotic system's strange attractor that initially separate slightly from each other will diverge exponentially in one direction while converge each other exponentially in another direction (Gollub, 2000).

\section{PREVIOUS CHAOS STUDIES OF EXCHANGE RATES}

Bask (1996) considered Swedish Kroner Vs Deutche Mark, ECU, US \$ and Yen. Data is daily observation from January 1986 to August 1995 (2409 points). By measuring the largest Lyapunov exponent, he found indication of deterministic chaos in all exchange rate series. In another study (Bask, 2002) for same exchange rate series using 1100 daily observations (from 17 May to 1991 to 31 August 1995) and employing bootstrap method, he found positive Lyapunov exponent.

Richards (2000) demonstrated fractal properties are characteristic of foreign exchange markets (country exchange rate, interest rates and interest rate differentials) across a broad range of countries (Australia, Canada, UK, Japan etc.). He also noted in financial markets, scaling symmetries are weak compared to strong scaling symmetries in physics.

Cecen et al. (1996) investigated the possibility of a low-dimensional chaotic attractor in hourly returns of spot exchange rate recorded by Money Market Services for the British pound, Deutschmark, the Swiss Franc and the Japanese Yen. They used percentage changes by taking logarithmic growth rates in hourly observations between $2^{\text {nd }}$ January 1986 and 15 July 1986 (which contained 3196 trading hours and is average of last five bid rates by fifty largest banks). They found that correlation dimension estimates do not converge to a stable value and does not support in favour of low dimensional chaotic dynamics, however, found largest Lyapunov exponents appear to be positive. Cecen et al. concluded that there is non-linear dependence in hourly exchange rate (kurtosis coefficient $>3$ ) but the nature of this non-linear dependence is far from being deterministic.

Schwartz and Yousefi (2003) investigated for low correlation dimension in a number of exchange rates and established low fractal dimension in DEM/USD, GBP/JPY, GBP/USD and JPY/USD exchange rate series. They used data starting 13 Feb 1973 to 1998 (around 6500 daily observations). Focusing on return they used first order differencing of data in log form.

\section{DATA GATHERING}

There are three issues here: how to deal with nonstationarity, the number of observations required, and the source of data.

Non-stationary is not a criteria in the mathematical definition of chaos. Researchers who have applied detrending techniques such as the log-difference or rate of return to filter raw non-stationary series into a stationary series, often concluded with indecisive results (Cecen et al., 1996, and Erkal, 1996). Application of log-difference to detrend the long term trend of a data series may destroy the nonlinear structure of the dynamics (Peters, 1991). An alternative detrending method, the log-linear method proposed by Barnett and Chen (1988) was found with certain success in the identification of chaotic dynamics within financial time series without the support of statistical tests. Schwartz et al. (2003) considered eight different ARMA models to remove linear dependency in the time series.

\$Can/\$US exchange rate is found to have less limitations than others with respect to suitability for chaotic analysis. There has been less intervention by monetary authority from both countries to regulate the exchange rate. In addition, the openness in the trading between two countries under the NAFTA agreement, proximity for labour and other asset mobilisation has removed the possibility of any long term trend in the exchange rate movement. Therefore detrending has not been applied in this study. 
Many early studies applying chaotic analysis to economic time series used too few observations to generate any meaningful results; for example Barnett and Chen (1988) used about 800 weekly data points and Peters (1992) only 480 monthly data points,. As these are too few observations to identify chaotic structures neither study can justify that they have identified true chaotic dynamics, even though they claimed the mathematical evidences of chaotic dynamics in their results. Those who took note of this issue adopted longer data length to conduct empirical tests. Mayfield and Mizrach (1992) use 19,027 data to test the S\&P 500 Index sampled at 20-second intervals with logdifferences and found a correlation dimension of 3.5. Cecen and Erkal (1996) took 3,191 data of foreign exchange rates with log-differences to test and failed to find evidence of chaos. Evidence from the physical sciences (e.g.Sprott and Rowlands (1995) is that 3981 observations are the minimum required. Daily exchange rate for the over 30 years of floating exchange rates meets the minimum criteria.

While it is increasingly common to base statistical analysis of financial time series on data sourced from major data vendors such as Reuters, and Datastream there is sometimes smoothing in this data.

Daily trading data on $\$$ Can $/$ US is available from number of different sources. In this study, we considered data obtained from pacific exchange (www.pacific.), Federal Reserve (www.federalreserve.gov/release) and data stream®. Though data is available from beginning of 1971 January from all the three sources, we ignored the data between $1^{\text {st }}$ of January 1971 and $12^{\text {th }}$ of February 1973 because of fixed rate system against gold standard. Weekends and holidays are neglected and the observations are assumed evenly spaced with one day trading interval.

In comparison of data obtained from Federal Reserve, Pacific commerce and Datastream between 1986 and 2003, we found maximum absolute differences are $0.74 \%$ and $1.45 \%$. The difference occurred most often in the fourth digit. Figure below shows the plot of difference between Federal Reserve and pacific commerce data.

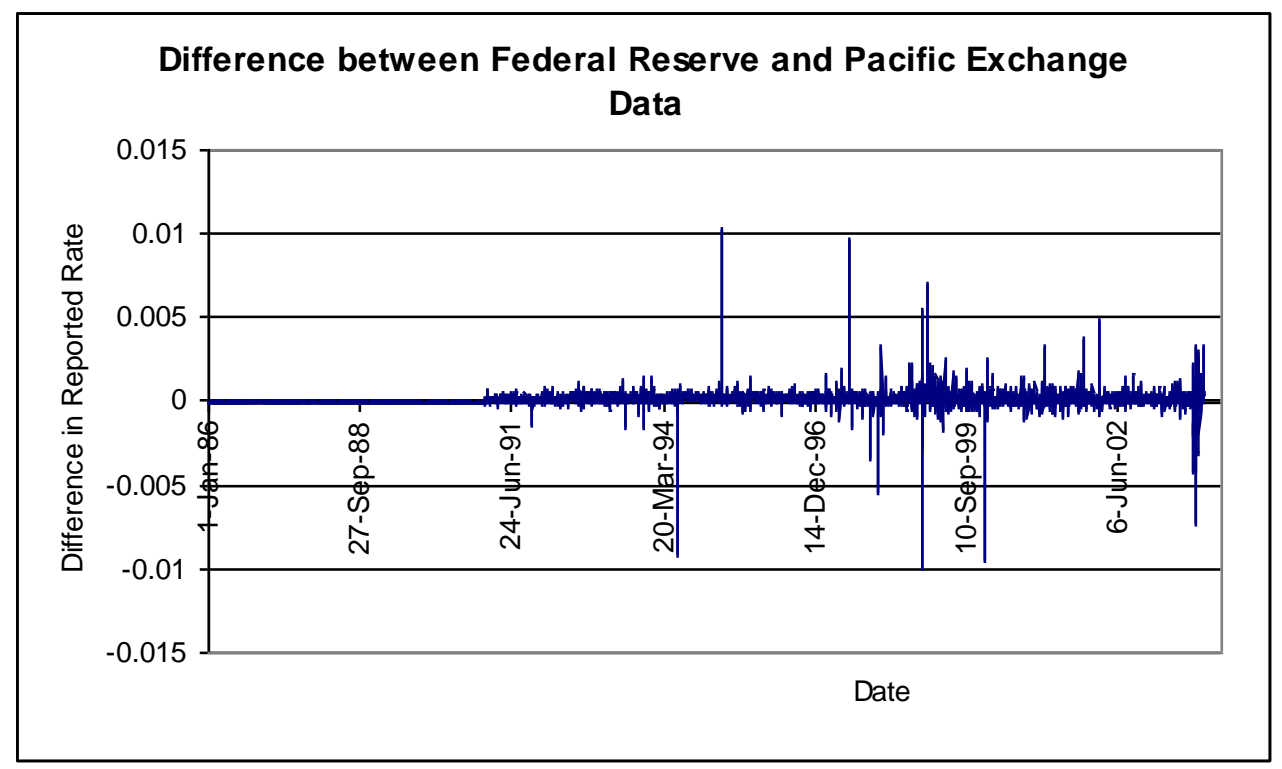

Figure 1: Difference in Reported Exchange Rate Between Federal Reserve and Pacific Exchange Data 


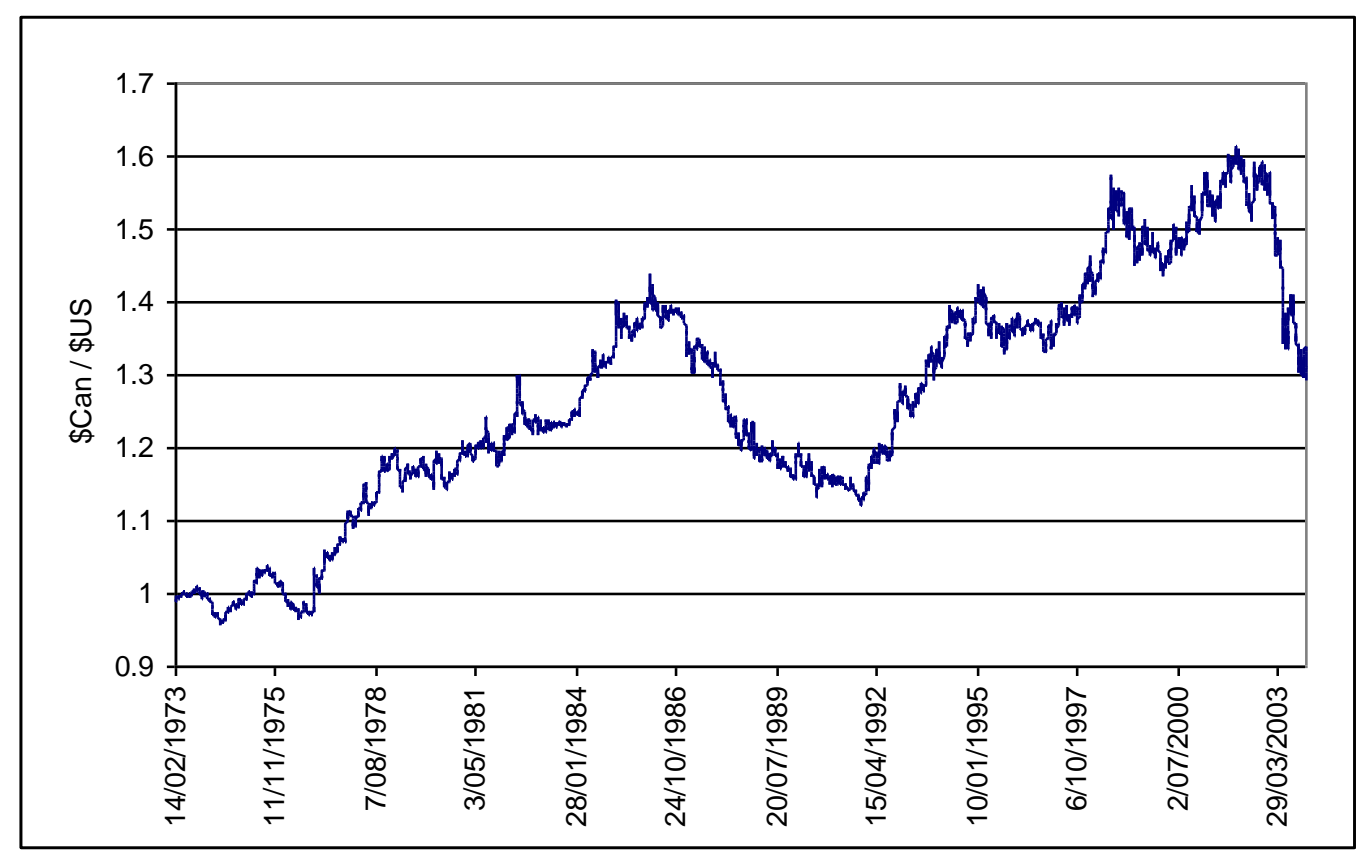

Figure 2: The \$C/\$US Exchange Rate 1973-2003

\section{CHAOTIC ANALYSIS OF THE \$C/\$US EXCHANGE RATE 1973-2003}

The identification of a chaotic structure for time series requires that noise must be of minimum level, but nonstationarity is not a requirement as demonstrated by Kennel (1997). For mathematical evidence of chaotic structure positive Lyapunov exponents and low correlation dimensions need to be found.

Knowledge of the dimension of a dynamical system reveals the order or complexity of the system. A correlation dimension factor is defined to obtain a quantitative measure of the dimension of the attractor, which is the number of variables required to specify its points accurately. Grassberger and Procaccia (1984) proposed a method to estimate the correlation dimension from the phase space patterns. The correlation dimension is derived from the correlation integral, which is a measure of spatial orientation of points on the strange attractor (Barnett and Chen, 1988). According to the Grassberger and Procaccia (1984) method, if the correlation integral C( $r, m)$ measures the fraction of total number of pairs $\left(\mathrm{x}_{\mathrm{i}}, \mathrm{x}_{\mathrm{i}+1}, \mathrm{x}_{\mathrm{i}+2}, . . \mathrm{x}_{\mathrm{i}+\mathrm{m}-1}\right)$ and $\left(\mathrm{x}_{\mathrm{j}}, \mathrm{x}_{\mathrm{j}+1}, \mathrm{x}_{\mathrm{j}+2}, . . \mathrm{x}_{\mathrm{j}+\mathrm{m}-1}\right)$ such that the distance between them is no more than $r$, then the correlation dimension is defined as:

$\mathrm{d}_{\mathrm{c}}=\lim (\mathrm{r} \rightarrow 0) \log \mathrm{C}(\mathrm{r}, \mathrm{m}) / \log (\mathrm{r})$

In the above limit equation, $\mathrm{C}(\mathrm{r}, \mathrm{m})$ is the correlation integral calculated for each embedded dimension by increasing the distance r. Figure 2 shows the correlation integrals curves for \$Can/\$US exchange rate series from 1973 to 2003 for increasing embedded dimension from 1 to 20 . Correlation dimension is calculated by finding the slope of each curve as $r \rightarrow 0$. As shown in figure 3, with the increasing embedding dimension, $d_{m}$ saturates to the correlation dimension of the attractor $d_{c}$. Saturation of correlation dimension for a large embedded dimension is indicative of determinism in the data.

One shortcoming in financial time series in applying chaos theory is the short time series or small data set available. Correlation dimension calculated from small data set is unreliable and should be interpreted cautiously. An estimation of minimum data requirement is $10^{2+0.4 \mathrm{~d}}$, where $\mathrm{d}$ is the least integral dimension containing the attractor (Tsonis, 1992, Sprott and Rowlands, 1995). For example, minimum data requirements for $\mathrm{d}=4 \mathrm{and} \mathrm{d}=5$ are 3,981 and 
10,000 respectively. The \$Can/\$US exchange rate series from 1973 to 2003 consists of 7754 observations and satisfies the above requirement for a correlation dimension less than four.

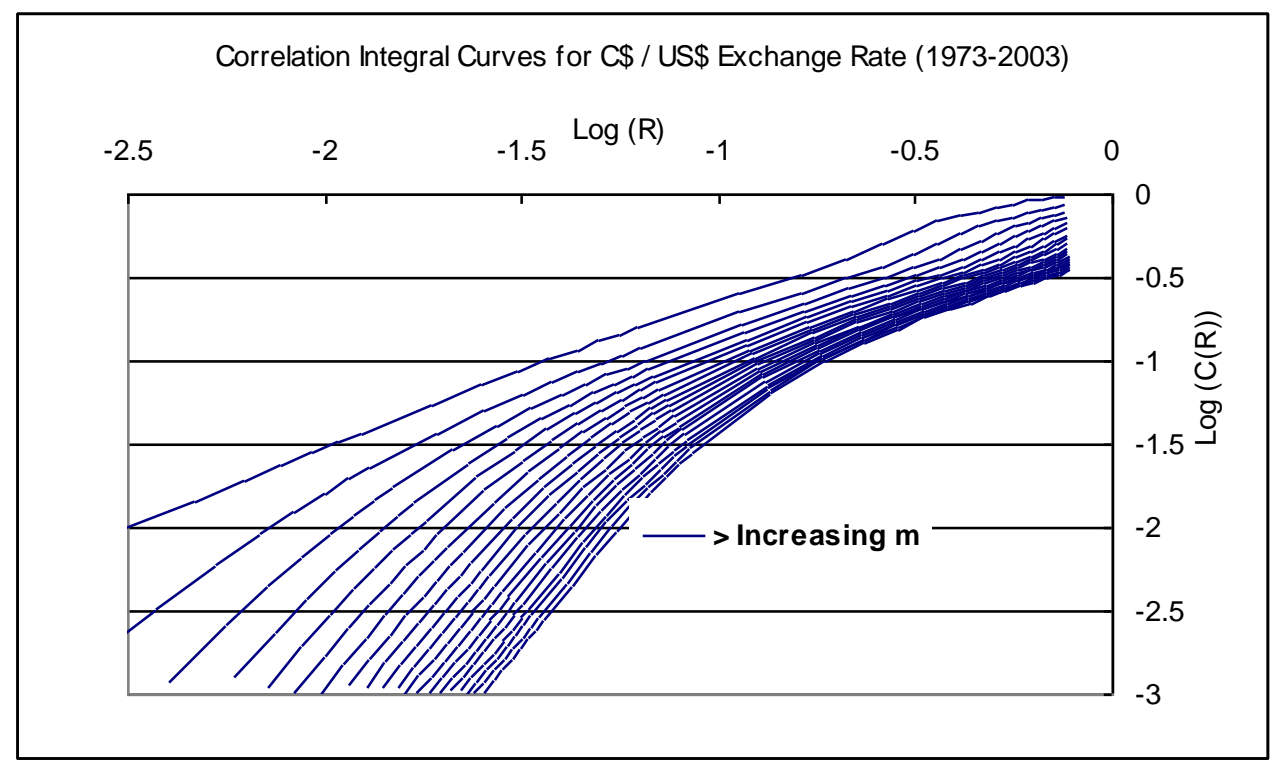

Figure 2: Correlation Integral Curves for \$Can/\$US Exchange Rate Series from 1973 to 2003

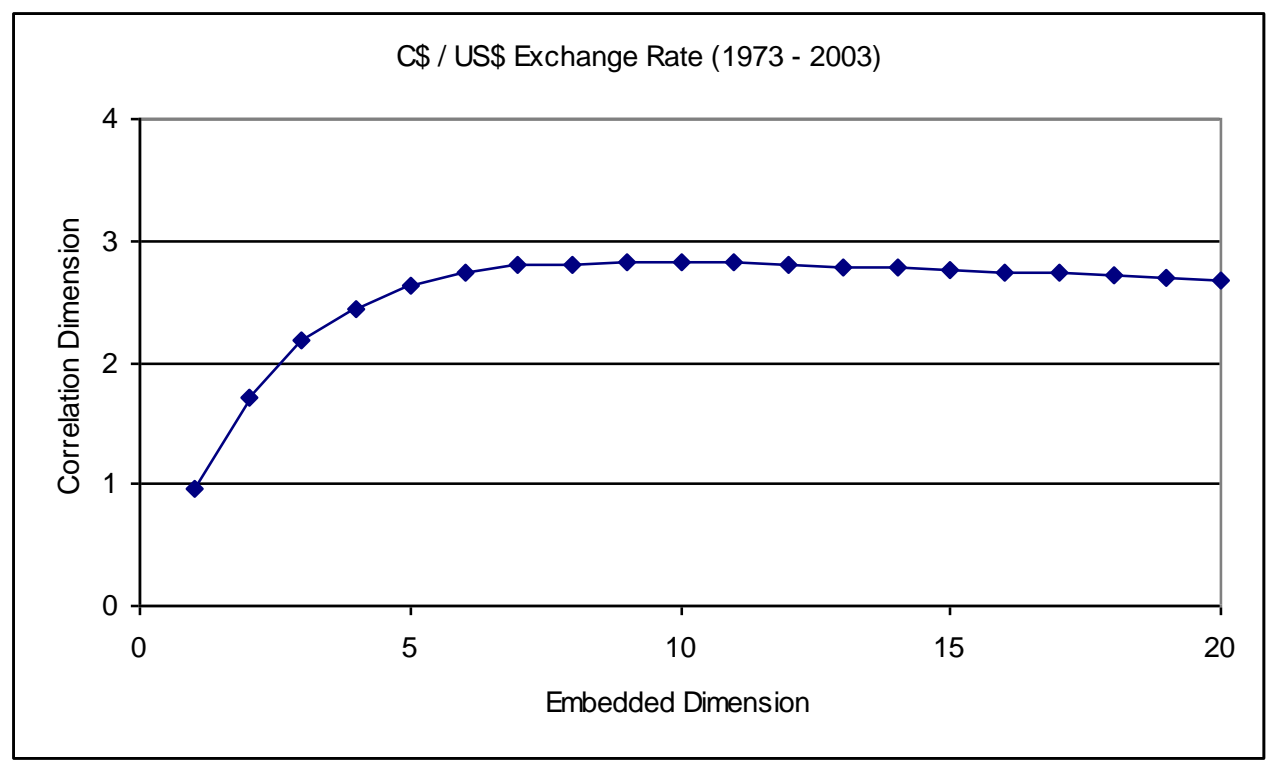

Figure 3: Correlation Dimension for \$Can/\$US Exchange Rate 1973 to 2003

Since 3981 data points (approximately 15 years data) are enough to calculate correlation dimension of less than four, 15 blocks of data were formed from January 1974 to December 2003. Each block starts on first day of trading in each year from 1974 to 1988. 


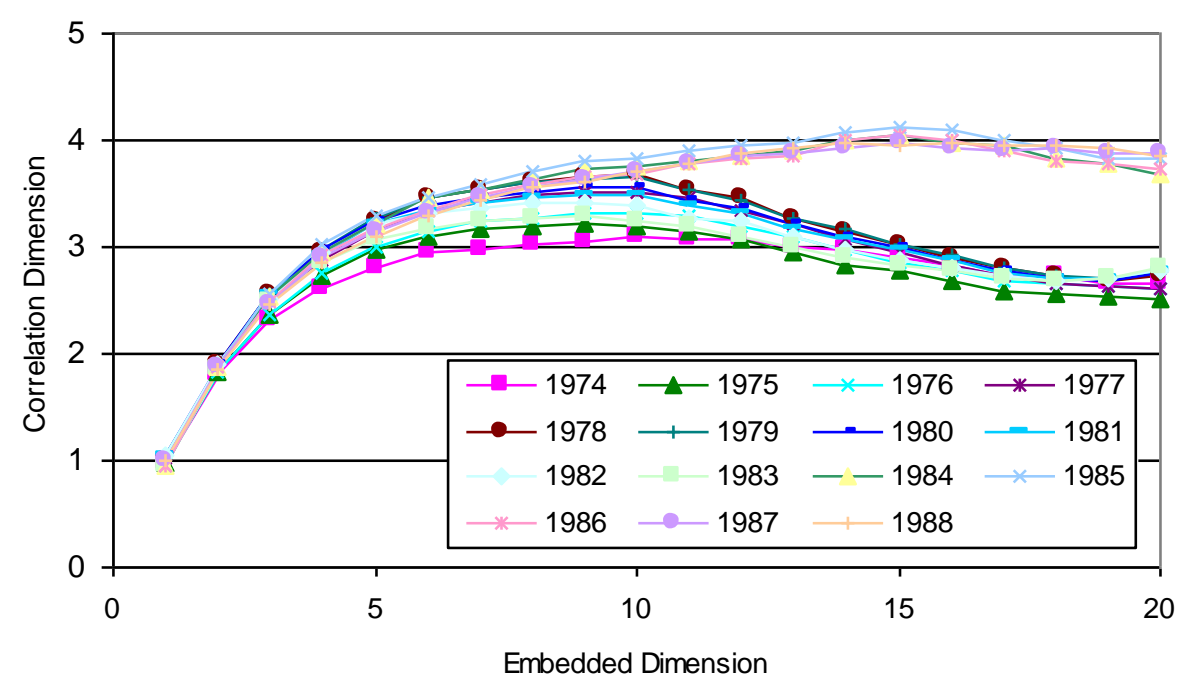

Figure 4: Evolution of Correlation Dimension of \$Can/\$US Since 1974

Figure 5 depicts the evolution of saturated correlation dimension since 1973. Correlation dimension of \$Can/\$US exchange series has changed since floating of exchange rate in 1973. Though it is not low-dimensional chaotic, it is less than 5 and the system is solvable.

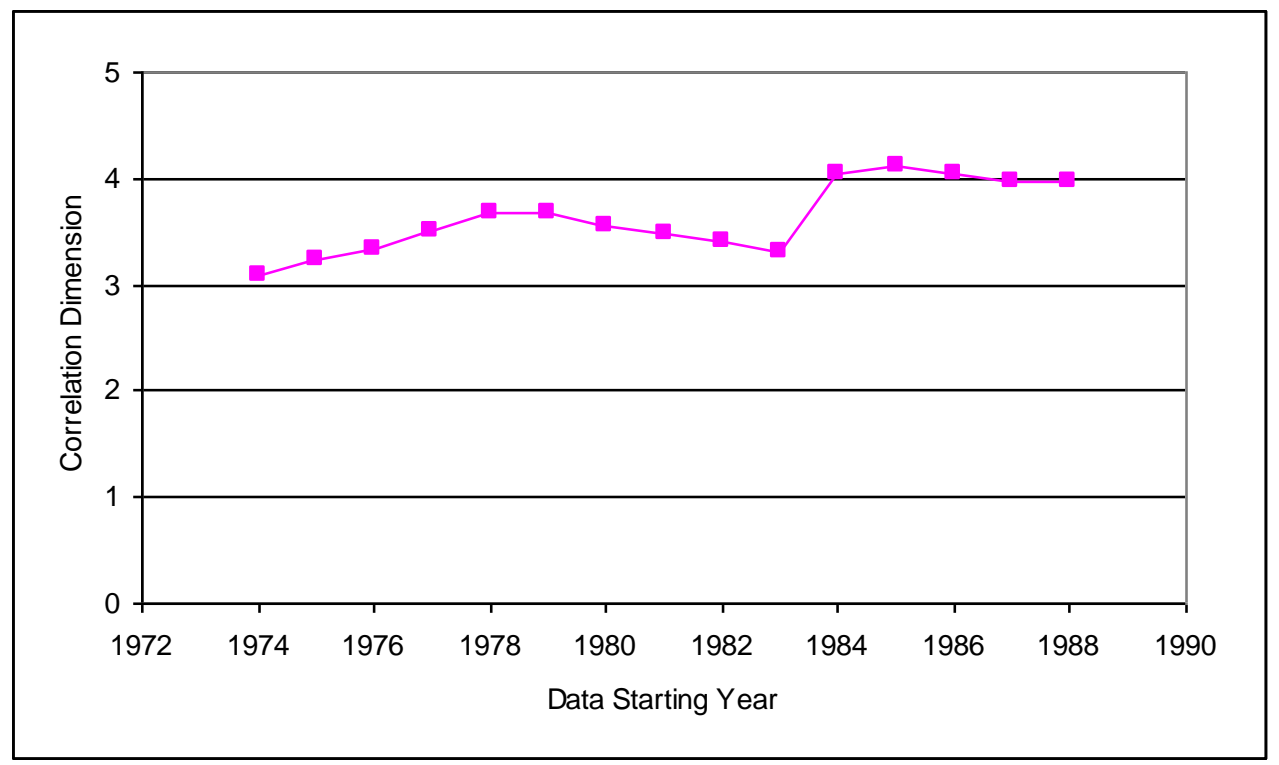

Figure 5: Evolution of Saturated Correlation Dimension Since 1973 in \$Can/\$US Exchange Data

Though floating of major exchange rates occurred in 1973, full floating of Australian \$ has happened only after 1986. Daily observations from 1988 to 2003 are enough for detecting correlation dimension lower than 4. In figure 6, the correlation dimension of \$Aus/\$US is compared with that found for the $\$ C$ an/ $\$$ US. It can be seen that the Canadian dollar series saturates more fully. 


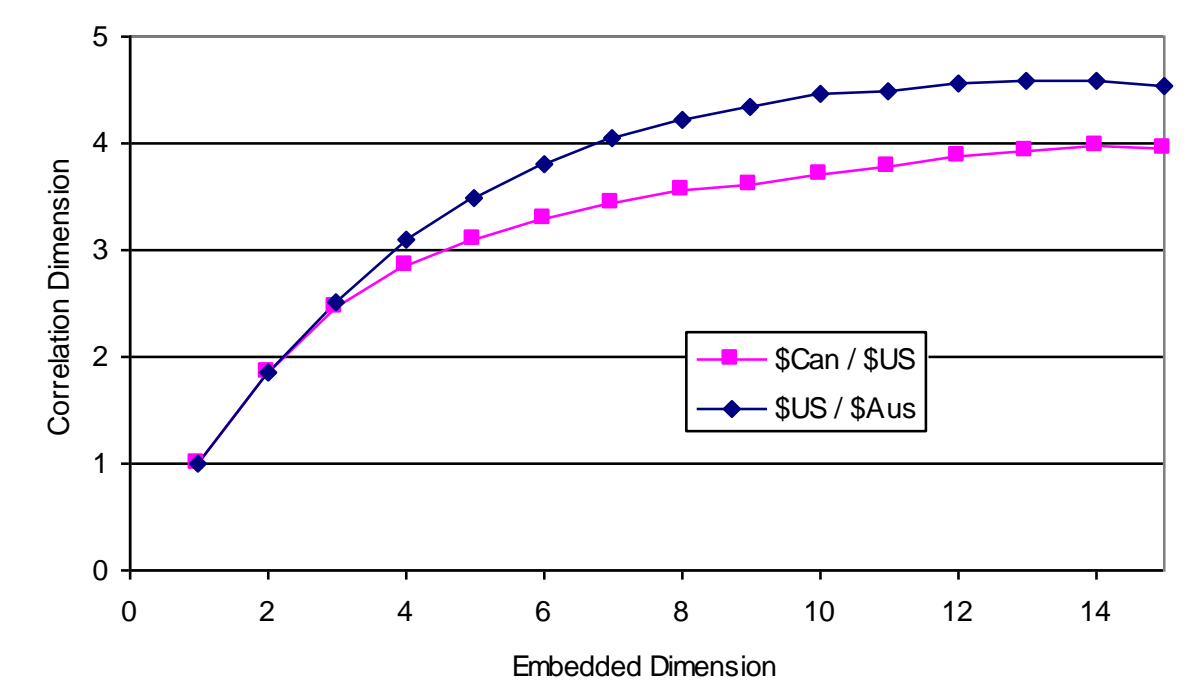

Figure 6: Comparison of Correlation Dimension of \$Aus/\$US and \$Can/\$US, Exchange Rate data from 1988 to 2003

A major source of chaos is the high sensitivity of the system output to the initial values of input and slight changes of system parameter values. Two trajectories on the attractor that initially separate slightly from each other will diverge exponentially in one direction while converging towards each other exponentially in another direction (Gollub, 2000). However, the solution paths will remain within a bounded set if the dynamic system is dissipative. By measuring separation of nearby points in phase space, the existence of chaos may be examined. The divergence can be measured by the Lyapunov exponents. The number of Lyapunov exponents is equal to the dimension of the underlying dynamic system. A positive exponent measures the exponential divergence of nearby points and a negative exponent measures the exponential convergence. Chaos exists only when the largest Lyapunov exponent is positive. A certain amount of data is required to calculate Lyapunov exponent reliably. This minimum requirement is same as the estimation for correlation dimension already made.

The Lyapunov exponent for the full series (1973 to 2003) is found to be 0.074. Figure 7 shows the evolution of largest positive Lyapunov exponent since 1973. Data blocks each consisting of 3981 data starting from first trading day of each year were considered.

We examined the $\$ \mathrm{Can} / \$ \mathrm{US}$ exchange rate series for sufficient conditions (low correlation dimension, positive Lyapunov exponent) for the existence of chaotic structure.. The correlation dimension of \$Can/\$US exchange rate series (1973-2003) was found to be 2.8. However, when 3981 blocks of observation were considered starting with the first trading day of each year since 1974, the correlation dimension is seen to have increased from 3.1 to 4.1 . The estimated correlation dimension is high predicting the system is complex. Increases in correlation dimension indicates the increasing complexity of international financial markets with globalisation, and development of new financial products (i.e., derivatives). The lack of saturation in the correlation dimension may also be due to insufficient length of the data sets because the Grassberger and Procaccia (1984) procedure relies on the assumption that the data set is of infinite length. \$Can/\$US correlation dimension for period 1988 to 2003 was 4.0 compared to 4.6 for \$US/\$Aus.

The Lyapunov exponent for the full series (1973 to 2003) was found to be 0.074 . When considered in blocks, the Lyapunov exponent has evolved from .087 (in 1974) to maximum of 0.145 (in 1980) and 0.120 for the last segment 1988-2003. That means predictability of $\$$ Can/ $\$$ US is limited to maximum of $8(=1 / 0.12)$ trading days if we use last 15 years of data (1988-2003) for non-linear prediction. 


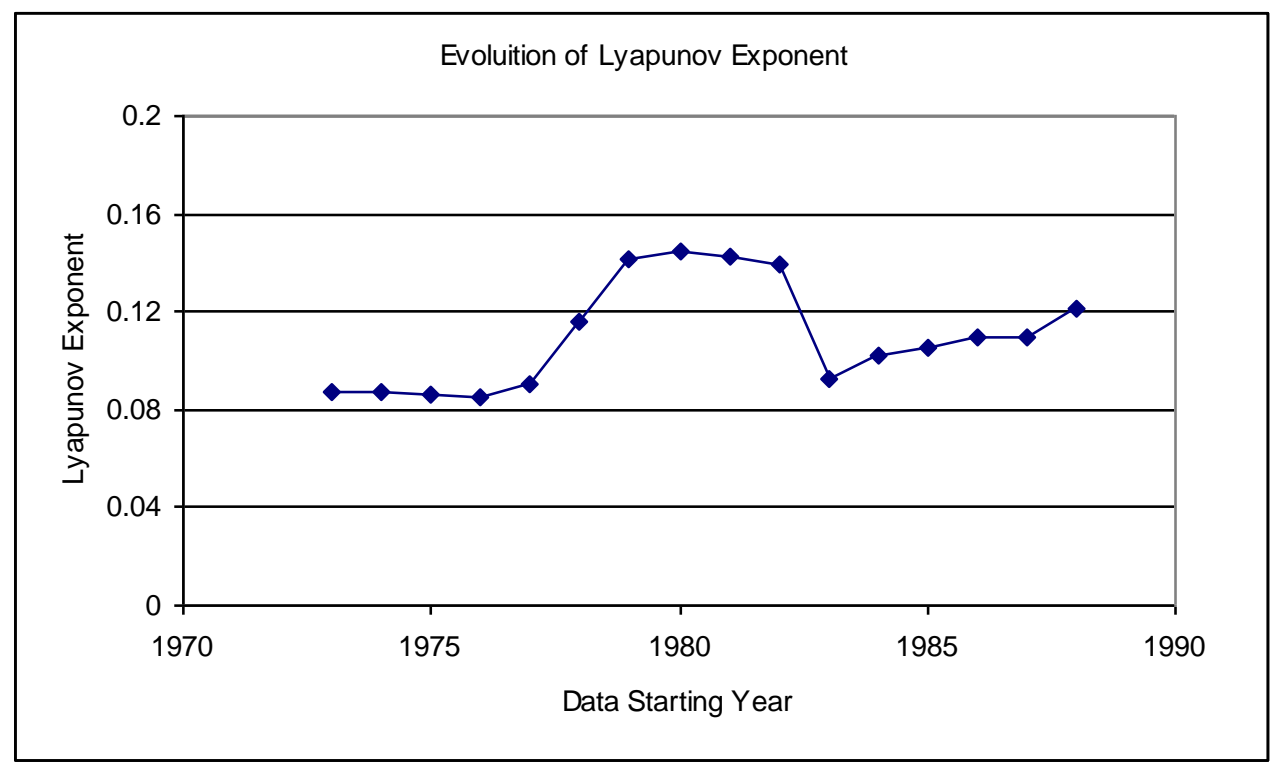

Figure 7: Evolution of Largest Positive Lyapunov Exponent of \$Can/\$US Exchange Rate Series (1973 - 2003)

\section{CONCLUSIONS}

We proposed that if we frame the "overshooting" hypothesis for exchange rate movements in terms of chaotic structure this may connect a theoretical view with practical substance. If we take the 1973-2003 period for the $\$ C / \$ U S$ exchange rate we do find sufficient conditions for chaotic structure. There is no evidence from the blocks of data tested that the Bank of Canada's intervention in the foreign exchange market has influenced the exchange rate away from its chaotic pattern. For shorter data sets the results show an increase in the correlation dimension towards the end of the period, but this may well be accounted for by the shorter length of data.

We compare the Canadian results with those for the \$A/\$us for the 1988 to 2993 period and find that of the two, the Canadian dollar offers results more fully aligned with the overshooting hypothesis.

\section{REFERENCES}

1. Aguilar, J. and S. Nydahl (1998). Central Bank Intervention and Exchange Rates: The case of Sweden, Working Paper, Sveriges Riksbank No 54.

2. Barnett, W. and P. Chen (1988). Deterministic chaos and fractal attractors as tools for nonparametric dynamical econometric inference: with an application to the divisia monetary aggregates, Mathematical and Computer Modeling, No.10, p. 275-296.

3. Bask, M. (2002). A positive Lyapunov exponent in Swedish exchange rates? Chaos, Solitons and Fractals, 14.

4. Beattie, Neil and Jean-Francois Fillion (1999). An Intraday Analysis of the Effectiveness of Foreign Exchange Intervention, Working Paper, 99-4, Bank of Canada.

5. Brock, W. A. and C. L. Sayers (1988). Is the Business Cycle Characterised By Deterministic Chaos?, Journal of Monetary Economics, 22, p.71-90.

6. Cecen A.A. and C. Erkal (1996). Distinguishing between Stochastic and Deterministic behaviour in foreign exchange rate returns: Further evidence, Economics Letters, 51, p. 323-329.

7. Chen, P. (1998). Empirical and Theoretical Evidence of Economic Chaos, System Dynamics Review, 4, No. 1-2, p. 81-108.

8. D'Souza, Chris (2000). A Market Microstructure Analysis of Foreign Exchange Intervention in Canada, Working Paper, 2002-16, Bank of Canada. 
9. Dominguez, K. (1998). Central bank intervention and exchange rate volatility, Journal of International Money and Finance, 12 (4).

10. Dornbusch, R. (1976). Expectations and Exchange Rate Dynamics, Journal of Political Economy, 84, No. 6, p. 1161-1176.

11. Gollub, J. P. and M. C Cross (2000). Chaos in space and time, Nature 404.

12. Grassberger, P. and I. Procaccia (1984). Dimensions and entropies of strange attractors form a fluctuating dynamics approach, Physics 13D.

13. Hung, J. (1997). Intervention Strategies and exchange rate volatility: a noise trading perspective, Journal of International Money and Finance, 16(5).

14. Laidler, David (1999). The Exchange Rate Regime and Canada's Monetary Order, Working Paper, 99-7 Bank of Canada.

15. LeBaron, B. (1994). Chaos and Nonlinear Forecastability in Economics and Finance, Philosophical Transactions of Royal Society of London, Series A, 348, p. 397-404.

16. Liu, T., C. W. J. Granger, and W. P. Heller (1992). Using the Correlation Exponent to Decide Whether an Economic Series is Chaotic, Journal of Applied Economics, 7, p. 525-539.

17. Murray, J., S. van Norden, and R. Vigfusson (1996). Excess Volatility and Speculative Bubbles in the Canadian Dollar: Real or Imagined?, Technical Report, No. 76, Bank of Canada.

18. Peters, E. (1991). Chaos and Order in the Capital Markets, John Wiley \& Sons Inc., New York.

19. Powell, J. (1998). A History of the Canadian Dollar, Bank of Canada.

20. Ramsey, J.B., S. L. Sayers, and P. Rothman (1990). The Statistical Properties of Dimension Calculations using Small Sets: Some Economic Applications, International Economic Review, 31, p. 991-1020.

21. Richards, G.R. (2000). The Fractal Structure of Exchange Rates: Measurement and Forecasting, Journal of International Financial Markets, 10, p. 163-180.

22. $\quad$ Rogoff, K. (1996). The Purchasing Power Parity Puzzle, Journal of Economic Literature, 34, p. 647-668.

23. Scheinkman, J.A. and B. LeBaron (1989). Nonlinear Dynamics and Stock Returns, Journal of Business, 62 , p. 311-337.

24. Schwartz, B. and S. Yousefi (2003). On Complex Behavior and Exchange Rate Dynamics, Chaos Solutions and Fractals, 18, p. 503-523. 